Internationale Forschungskooperation mit der Slovakei:

\title{
Poröse Sinterkeramikformstoffe mit katalytischer Wirkung durch thermische Behandlung von mit Zeolithen hochgefüllten Schaumstoffen auf der Basis von Recyclaten
}

\author{
Martin Lorko, Peter Oravec, Karl-Heinz Schmidt, Hans-Dieter Hunger, Gerhard Behrendt
}

\section{Einleitung}

Poröse Sinterkeramikwerkstoffe mit unterschiedlichen Porenstrukturen werden heute in vielen Bereichen der Wirtschaft eingesetzt, z. B. als Trennmembranen, als Katalysatorträger, als Trägermaterialien in der Biotechnologie. Dabei sind zwei Arten von Sinterkeramiken zu unterscheiden: zum einen wabenförmige Sinterkeramiken und zum anderen schaumförmige Sinterkeramiken. Die wabenförmigen, oft als Strang hergestellten Sinterkeramiken, werden in immer größerem Maße als Trennmedien, z. B. in der Wasser- und Abluftreinigung, verwendet. Sie sollen hier jedoch nicht weiter betrachtet werden. Die schaumförmigen Sinterkeramiken mit unterschiedlich großer Zellgröße bzw. Zellgrößenverteilung werden als Träger oder als Hochtemperaturisolation eingesetzt. Sie werden nach zwei verschiedenen Technologien hergestellt: zum einen durch die Füllung der keramischen Massen mit organischem Material, das beim Brennprozess verdampft wird, und zum anderen durch Beschichten von Schaumstoffen mit Keramikschlämmen und deren Brennen, wobei während des Brennens das organische Schaummaterial verdampft wird.

An der Technischen Universität Kosice werden Untersuchungen mit schalldämmenden Materialien für Verbrennungskraftmaschinen durchgeführt, für die verschiedene Stoffe eingesetzt und mittels schalltechnischer Messungen untersucht werden. Weiterhin stehen in der Slovakischen Republik natürliche Zeolithe zur Verfuigung, die bereits bei relativ niedrigen Temperaturen zu sintern beginnen. An der Technischen Fachhochschule Wildau wurden im Rahmen von Forschungskooperationen mit der mittelständischen Wirtschaft Hartschaumstoffsysteme speziell für einen hohen Füllstoffgehalt auf der Basis von Recyclaten entwickelt. Aus diesen RecyclatSystemen und den Zeolithen der slovakischen Partnerinstitution wurde ein Verfahren zur Herstellung von Sinterkeramikwerkstoffen entwickelt (1).

In einem vom Internationalen Büro des BMBF geförderten Forschungsvorhaben wurden in den Jahren 2000 bis 2003 Untersuchungen zur Herstellung von keramischen Schaumstoffen und ihrer Charakterisierung hinsichtlich der Schalldämmung sowie der Adsorption von Schadstoffen auf ihrer Oberfläche durchgeführt. Die gemeinsam erhaltenen Ergebnisse sollen hier kurz dargestellt werden.

\section{Materialien und Methoden}

\subsection{Verwendete Materialien}

\subsubsection{Zeolithe}

Als keramisches Basismaterial wurden in der Slovakischen Republik natürlich vorkommende Zeolithe der Lagerstätte $\mathrm{Ni} \bullet$ ný Hrabovec verwendet (Abbildung 1).

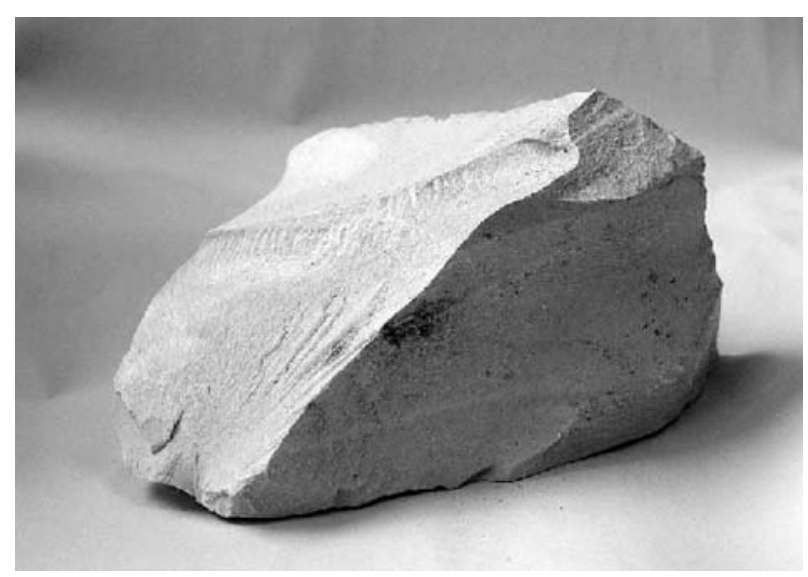

Abb. 1: Zeolith aus der Grube Ni •ný Hrabovec (Originalzustand)

Die Zusammensetzung der Mineralien ist

Clinoptilolit 60,0\% Plagioklas $5,2 \%$ Quartz $3 \%$ Cristobalit $\quad 8,6 \%$ amorphe Phase $8,0 \%$ Biotit $1 \%$

Die chemische Zusammensetzung ist:

$\begin{array}{lrlr}\mathrm{Al}_{2} \mathrm{O}_{3} & 11,5-13,10 \% & \mathrm{CaO} & 2,70-5,20 \% \\ \mathrm{~K}_{2} \mathrm{O} & 2,2-3,40 \% & \mathrm{Fe}_{2} \mathrm{O}_{3} & 0,70-1,90 \% \\ \mathrm{MgO} & 0,6-1,20 \% & \mathrm{MnO} & 0,12 \% \\ \mathrm{FeO} & 0,39 \% & \mathrm{Na}_{2} \mathrm{O} & 0,20-1,30 \% \\ \mathrm{SiO}_{2} & 65,0-71,30 \% & \mathrm{TiO}_{2} & 0,10-0,30 \%\end{array}$

Die Ionenaustauschereigenschaften sind:

Austauscherkapazität:

$\mathrm{Ca}^{2+} \quad 0,64-0,98 \mathrm{~mol} / \mathrm{kg}$

$\mathrm{K}^{+} \quad 0,22-0,45 \mathrm{~mol} / \mathrm{kg}$

$\mathrm{Mg}^{2+} 0,06-0,19 \mathrm{~mol} / \mathrm{kg}$

$\mathrm{Na}^{+} \quad 0,01-0,19 \mathrm{~mol} / \mathrm{kg}$

Gesamtaustauscherkapazität:

Erweichungstemperatur: $\quad 1260^{\circ}$

Dichte:

Schmelztemperatur:

$2200-2440 \mathrm{~kg} / \mathrm{m}^{3}$

$1340{ }^{\circ} \mathrm{C}$

Das Schmelzverhalten der Zeolithe wurde durch die Bestimmung der Schmelzpeaks in der DTA bestimmt und zeigt folgendes Ergebnis: 


\section{Schmelzpeak $832-956{ }^{\circ} \mathrm{C}$ \\ 2. Schmelzpeak $1087-1123^{\circ} \mathrm{C}$ \\ 3. Schmelzpeak $1335-1378{ }^{\circ} \mathrm{C}$}

Durch den niedrigen Schmelzbeginn konnte für die Herstellung der Keramikschäume eine niedrige Sintertemperatur gewählt werden.

Zur Einarbeitung in die schäumfähigen Gemische wurden verschiedene Mahlgutqualitäten mit unterschiedlichen Teilchengrößen verwendet. Das eingesetzte Mahlgut hatte folgende mittlere Teilchengrößen
MGZ 3
$2 \mathrm{~mm}$,
MGZ 7
$0,5 \mathrm{~mm}$,
MGZ 11
$0,1 \mathrm{~mm}$.

Die Abbildungen 2 bis 4 zeigen die Teilchengrößenverteilungen der verwendeten Mahlguiter.

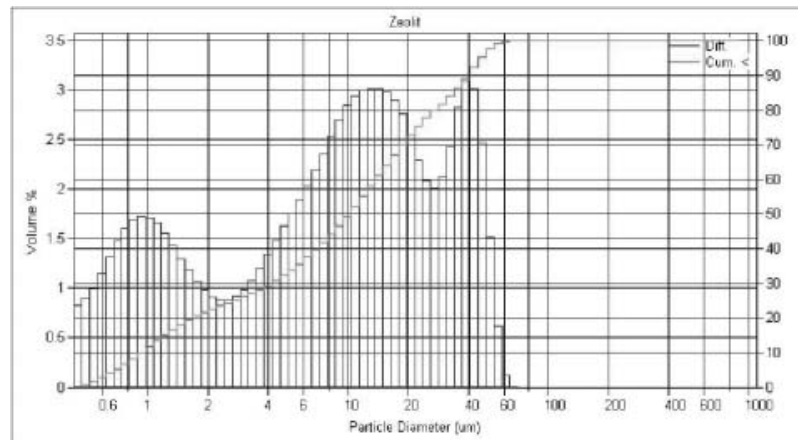

Abb. 2: Korngrößenverteilung des verwendeten Zeolith-Pulvers

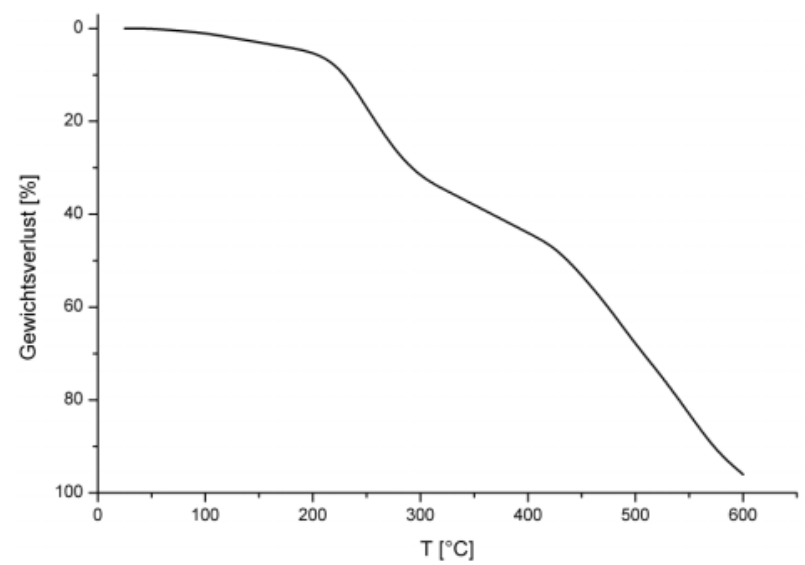

Abb. 3: TGA-Kurve des Polyurethanhartschaumstoffs auf der Basis von P-185

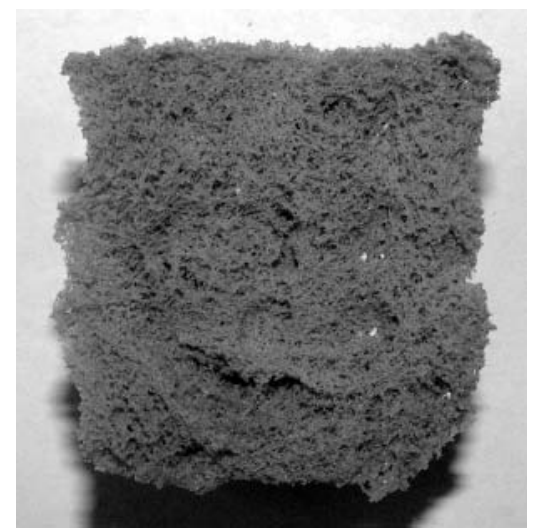

Abb. 4: Sinterkeramikschaumstoff 51 nach dem Brennprozess
Das Mahlgut wurde mit der jeweiligen Polyolkomponente auf der Brabender ${ }^{\circledR}$ Labstation mit Knetervorsatz 30 Minuten homogenisiert und in dieser Form mit den weiteren Formulierungsbestandteilen versetzt und verschäumt.

\subsubsection{Herstellung der Hartschaumstoffsysteme}

Als Hartschaumstoffsysteme wurden Zwei-Komponenten-Systeme auf Polyurethanbasis eingesetzt. Die Formulierungen wurden mit Polyolen entwickelt, die durch Solvolyse von Polyurethan-Weichschaumstoffen (RP-V02) (1) bzw. durch Glykolyse von Polyethylenterephthalat (P185) (2) hergestellt wurden.

Die Basisformulierungen sind in der folgenden Tabelle zusammengestellt:

\begin{tabular}{|l|r|r|}
\hline Komponente/Formulierung & $R P-213$ & $R P-238$ \\
\hline Polyol RP-V02 & 172,5 & \\
Polyol P-185 & & 153,7 \\
Dimethylethanolamin & 0,5 & 0,6 \\
Dimethylcyclohexylamin & & 0,3 \\
Polycat NP-40 & 0,3 & 0,2 \\
Tegostab B- & 0,4 & 0,5 \\
Wasser & 4,5 & 4,5 \\
\hline Lupranat M 20 A® & 202,5 & 200,0 \\
\hline Ruihrzeit im Handansatz (s) & 10 & 10 \\
Startzeit (s) & 16 & 18 \\
Steigzeit (s) & 68 & 72 \\
Abbindezeit (s) & 90 & 94 \\
\hline
\end{tabular}

Tab. 1: Basisformulierungen für PUR-Hartschaumstoffe auf der Basis von PUR-Recyclatpolyol (RP-V02) und aromatischem Polyesterpolyol (P-185)

Bei den gröberen Mahlgütern wurden 100, 200 und 300 Gew.-Prozent bezogen auf die A-Komponente, bei den feineren Mahlguitern 250, 400, 500 und 600 Gew.-Prozent bezogen auf die A-Komponente verwendet. Die Schaumstoffe wurden zunächst im Handansatz in 1 LiterBechern freiverschäumt hergestellt. Für die Brennversuche wurden die Schaumstoffe in einer 2 Liter-Aluminiumform von $10 \times 10 \times 20 \mathrm{~cm}$ unter Druck hergestellt. Aus den Schaumstoffblöcken wurden nach einer einwöchigen Nachhärtungszeit drei Prüfkörper von 5 x 5 x $5 \mathrm{~cm}$ solcherart herausgeschnitten, dass jeweils $2,5 \mathrm{~cm}$ der Außenzonen als Verschnitt entstanden und ausschließlich die Innenzonen für die Versuche verwendet wurden. An diesen Schaumstoffen wurden die Rohdichten und eine mittlere Zellgröße bestimmt.

\subsection{Ausbrennversuche}

Die Schaumstoffwürfel wurden einer thermischen Behandlung in einem Carbolite-Hochtemperatur-Muffelofen Typ 2408 unterworfen. Die thermische Behandlung wurde mit den vorher auf der Basis von DTA-Messungen bestimmten Abbautemperaturen festgelegt. Die Temperaturprogramme berücksichtigen insbesondere die maximalen Abbaugeschwindigkeiten der organischen Komponente, die nach TGA/DTA folgende Maxima aufweist (Beispiel RP-238 ohne Füllstoff, die Formulierung RP-213 weicht nur unwesentlich von diesen Werten ab): 
1. Maximum bei $249^{\circ} \mathrm{C}$

2. Maximum bei $432^{\circ} \mathrm{C}$

3. Maximum bei $539^{\circ} \mathrm{C}$

Der Gewichtsverlust wurde über die TGA bestimmt (siehe Abbildung 5). Der maximale Gewichtsverlust erfolgt im Bereich oberhalb $420^{\circ} \mathrm{C}$.

Die zum Ausbrennen Abb. 5: Sinterkeramikschaumstoff 36 verwendeten Tempe- nach dem Brennprozess raturprogramme wur-

den so ausgelegt, dass bei $600^{\circ} \mathrm{C}$ noch ein Rest an organischem Material zur Bindung der Zeolithe vorhanden sein soll; in Tabelle 2 sind die im wesentlichen angewandten Temperaturprogramme dargestellt:

\begin{tabular}{|l|r|r|r|r|}
\hline $\begin{array}{l}\text { Temperaturbereich/ } \\
\text { Programm }\end{array}$ & TP1 & TP3 & TP6 & TP9 \\
\hline $20-250^{\circ} \mathrm{C}$ & $3 \mathrm{~h}$ & $2 \mathrm{~h}$ & $2 \mathrm{~h}$ & $2 \mathrm{~h}$ \\
\hline $250-500^{\circ} \mathrm{C}$ & $2 \mathrm{~h}$ & $2 \mathrm{~h}$ & $1,5 \mathrm{~h}$ & $2 \mathrm{~h}$ \\
\hline $500-680^{\circ} \mathrm{C}$ & $2 \mathrm{~h}$ & $1,5 \mathrm{~h}$ & $2 \mathrm{~h}$ & $1,5 \mathrm{~h}$ \\
\hline $680-900^{\circ} \mathrm{C}$ & $4 \mathrm{~h}$ & $3 \mathrm{~h}$ & $3 \mathrm{~h}$ & $1,5 \mathrm{~h}$ \\
\hline $900-1050^{\circ} \mathrm{C}$ & & & $3 \mathrm{~h}$ & $3 \mathrm{~h}$ \\
\hline $900-1250^{\circ} \mathrm{C}$ & $4 \mathrm{~h}$ & & & \\
\hline
\end{tabular}

Tab. 2: Beispiele für Temperaturprogramme der Ausbrennversuche

Weitere Beispiele fuir individuelle Ausbrennprogramme werden unter Punkt 5 ,Diskussion der Ergebnisse“ unter Bezugnahme auf die damit erhaltenen Keramikschaumstoffe dargestellt. Nach dem Ende des Temperaturprogramms wurden der Ofen mit dem Material uiber Nacht heruntergekuihlt und das Material am folgenden Tag entnommen (Abkühlzeit 14 Stunden).

\subsection{Charakterisierung der Keramikschaumstoffe}

Die nach dem Brennvorgang erhaltenen Materialien wurden hinsichtlich ihrer Farbe und ihrer visuellen Erscheinung geprüft. Weiterhin wurde die Dimensionsveränderung (Schrumpfung) gemessen. Die Offenzelligkeit wurde durch die aufgenommene Menge an Wasser (iiber Dichtemessungen) bestimmt. Nach dieser Methode wurde ein Offenzelligkeitsgrad von $>97 \%$ bei allen Keramikschaumstoffen ermittelt.

Die Schrumpfung der Keramikschaumstoffe wurde durch Ausmessen mit einer Schublehre und mit Bezug auf die Ausgangsdimensionen bestimmt; bei einer Schrumpfung unter $10 \%$ wurden die Keramikschaumstoffe einem Testprogramm zur Ermittlung der Schalldämmung und der Adsorption von Gasen unterworfen.

Die Schalldämmung wurde mit einem Modul-Schallpegel-Analysator „Investigator“ mit BZ7210 sowie einem Uni-Gain-DetaShear-Beschleunigungsaufnehmer $1 \mathrm{pC} / \mathrm{ms}-2$ von Brüiel \& Kjaer gemessen. Dazu wurde folgende Versuchsanordnung verwendet: Die Schadstoffadsorption wurde an einem $50 \mathrm{~mm}$ langen Material in einem Abgasstrom eines Dieselmotors (Skoda 120 bzw.
Skoda Favorit $136 \mathrm{~L}$ ) in einem $50 \mathrm{~mm}$ Abgasrohr bestimmt, indem die Zusammensetzung des Abgasstromes bei konstant laufender Verbrennungskraftmaschine vor und nach dem Adsorptionsmaterial über 24 Stunden mittels GC-MS (Hewlett-Packard GC Typ 5890, MS Typ 5970) sowie die an Aktivkohle absorbierten polycyclischen Kohlenwasserstoffe (PAK) mittels HPLC bestimmt wurden. Aus der Differenz der Schadstoffe im Gasstrom vor und nach der Passage des Filtermaterials wurden der Adsorptionsgrad uiber 24 Stunden errechnet und eine Adsorptionskurve ermittelt.

\section{Ergebnisse}

\subsection{Herstellung von Keramikschaumstoffen nach dem Verfahren des direkten Ausbrennens gefiullter PUR-Hartschaumstoffe}

Keramikschaumstoffe wurden mit unterschiedlichen Gehalten an Zeolithen in der Polymermatrix (Anteil zwischen 22 und 50 Gew.-Prozent) unterschiedlichen Temperaturen und Temperaturprogrammen unterworfen. Die Schaumstoffe wurden aus aromatischen Polyesterpolyolen (auf PET-Basis durch Umesterung in einem Glykolgemisch nach (2) hergestellt) bzw. aus Recyclatpolyolen auf der Basis von PUR-Schaumstoffen (3), in die durch Kneten die erforderliche Menge an Zeolithpulver eingearbeitet worden war, in Gegenwart von tertiären Aminen als Katalysatoren, Wasser als Treibmittel und einem Silikonpolyether als Stabilisator im direkten Freischäumverfahren bzw. im Druckschäumverfahren in der geschlossenen Form hergestellt (siehe Tabelle 1). Die Knettechnik zur Herstellung der Premixes und daraus der A-Komponente des Polyurethan-Systems erwies sich als der günstigste Weg zur Herstellung der hochgefuillten Hartschaumstoffe. Das Polyol hatte dabei auf die Verarbeitungseigenschaften wenig Einfluss, da sich die Viskositäten der beiden ausgewählten Polyole bei $25^{\circ} \mathrm{C}$ nur wenig unterschieden. Wasser als Treibmittel wurde gewählt, um ein hohes Maß an Offenzelligkeit zu gewährleisten. Darüber hinaus ist Wasser das umweltfreundlichste Treibmittel und erzeugt beim Schäumprozess aus der Umsetzung mit dem Polyisocyanat Kohlendioxid, das langsam aus den Zellen diffundiert.

Eine erste Serie von gefüllten Hartschaumstoffen wurde mit 22 bis 25 Gew.-Prozent Zeolith hergestellt (Versuche 5, 8, 9). Diese Schaumstoffe wurden in einer ersten Ausbrennreihe verschiedenen Temperaturprogrammen unterworfen: Der Hartschaumstoff aus Versuch 5 mit 24 Gew.-Prozent Zeolith wurde schnell auf $900^{\circ} \mathrm{C}$ erwärmt und drei Stunden bei dieser Temperatur gehalten. Anschließend wurde eine Sinterzeit von vier Stunden bei $1150^{\circ} \mathrm{C}$ gewählt. Im Ergebnis wurde ein stark geschrumpftes Produkt mit sehr geringer Festigkeit erhalten, das für die weiteren Untersuchungen ohne Nachbehandlung nicht geeignet war. Es erfolgte eine weitere Sinterung des keramischen Produktes bei $1200^{\circ} \mathrm{C}$ über fuinf Stunden. Das fuihrte zu einem teilweisen Verschmelzen der Schaumstoffzellen, so dass die Sintertemperatur zu hoch eingestellt worden war. 
Mit dem Hartschaumstoff aus Versuch 8 mit 24,5 Gew.Prozent Zeolith wurde der Versuch wiederholt, jedoch wurde das Temperaturprogramm folgendermaßen eingestellt: Aufheizrate 30 Minuten auf eine Temperatur von $800^{\circ} \mathrm{C}$, Ausbrennen bei $800^{\circ} \mathrm{C}$ innerhalb einer Stunde, Sintervorgang bei $1000^{\circ} \mathrm{C}$ uiber sechs Stunden. Das Ergebnis war ein feinporiger keramischer Schaumstoff mit einer guten Festigkeit, jedoch mit einer Schrumpfung von $36 \%$ in der Länge. Der Versuch wurde mit dem Hartschaumstoff aus Versuch 9 mit 24 Gew.-Prozent Zeolith wiederholt. Es wurde jedoch eine weitere Variante eines Temperaturprogramms verwendet: Zunächst wurde der Schaumstoff innerhalb von drei Stunden auf $380^{\circ} \mathrm{C}$ erwärmt, danach innerhalb einer Stunde auf $500^{\circ} \mathrm{C}$, danach innerhalb von zwei Stunden weiter auf $920^{\circ} \mathrm{C}$ und schließlich innerhalb weiterer zwei Stunden auf $1150^{\circ} \mathrm{C}$. Als Ergebnis wurde ein stark geschrumpfter Keramikschaumstoff mit geringer Festigkeit erhalten.

In einer zweiten Versuchsserie wurden 35 Gew.-Prozent Zeolith in das schaumfähige Gemisch eingearbeitet und die Ausbrennversuche weitergefuihrt, um die Ausbrennbedingungen weiter zu optimieren (Versuche 2, 28, 41, 51, 52, 72). Die Temperaturprogramme wurden weiter variiert:

\begin{tabular}{|l|r|r|r|r|r|r|}
\hline Nr. & 2 & 28 & 41 & 51 & 52 & 71 \\
\hline 1. Schritt $\left({ }^{\circ} \mathrm{C} / \mathrm{h}\right)$ & $900 / 3$ & $380 / 3$ & $800 / 2$ & $800 / 2,5$ & $1000 / 6$ & $800 / 2$ \\
\hline 2. Schritt $\left({ }^{\circ} \mathrm{C} / \mathrm{h}\right)$ & $1150 / 4$ & $500 / 1$ & $1000 / 6$ & $1000 / 1$ & & $1000 / 1$ \\
\hline 3. Schritt $\left({ }^{\circ} \mathrm{C} / \mathrm{h}\right)$ & & $920 / 3$ & & $1100 / 2$ & & $1100 / 4$ \\
\hline 4. Schritt $\left({ }^{\circ} \mathrm{C} / \mathrm{h}\right)$ & & $1150 / 2$ & & & & \\
\hline
\end{tabular}

Tab. 3: Temperaturprogramme für die Ausbrennversuche von Hartschaumstoffen mit 35 Gew.-Prozent Zeolith

Das beste Ergebnis wurde beim Versuch 51 erhalten: ein Keramikschaumstoff mit einer Schrumpfung von $11 \%$, einer sehr feinen Struktur (Zellgröße zwischen 0,4 und $1,8 \mathrm{~mm}$ ) und einer ausreichenden Festigkeit, so dass eine Weiterverarbeitung (Formgebung) möglich ist. Bei der Anwendung eines Temperaturprogramms wie im Versuch 52 wird bei gleicher Zusammensetzung des Schaumstoffs eine wesentlich höhere Schrumpfung und eine deutlich gröbere Zellstruktur festgestellt.

In einer dritten Serie wurden die Hartschaumstoffe mit 44 bis 47 Gew.-Prozent Zeolith hergestellt und wiederum dem Brennprozess bei unterschiedlichen Temperaturen unterworfen. Ausgewählt wurden die Schaumstoffe der Versuche 16 und 36 (45 Gew.-Prozent Zeolith). Diese wurden jeweils zwei unterschiedlichen Temperaturprogrammen unterworfen:

\begin{tabular}{|l|r|r|r|r|}
\hline Nr. & 16 & 16 & 36 & 36 \\
\hline 1. Schritt $\left({ }^{\circ} \mathrm{C} / \mathrm{h}\right)$ & $380 / 3$ & $350 / 2$ & $380 / 3$ & $350 / 2$ \\
\hline 2. Schritt $\left({ }^{\circ} \mathrm{C} / \mathrm{h}\right)$ & $500 / 2$ & $600 / 2$ & $500 / 2$ & $600 / 3$ \\
\hline 3. Schritt $\left({ }^{\circ} \mathrm{C} / \mathrm{h}\right)$ & $920 / 2,5$ & $1150 / 4$ & $920 / 2$ & $1150 / 4$ \\
\hline 4. Schritt $\left({ }^{\circ} \mathrm{C} / \mathrm{h}\right)$ & $1150 / 1$ & & $1150 / 2$ & \\
\hline
\end{tabular}

Tab. 4: Ausbrennprogramme der Hartschaumstoffe mit 45 Gew.-\% Zeolith

Die Hartschaumstoffe mit höherem Gehalt an Zeolithen (Versuche 16 und 36) wiesen trotz unterschiedlicher Ausbrennprogramme eine deutlich schlechtere Festig- keit als die Schaumstoffe mit 35 Gew.-Prozent Zeolith auf. Außerdem war überraschenderweise die Schrumpfung wieder größer als im Falle der Schaumstoffe mit ca. 35 Gew.-Prozent Zeolith. Die aus den Versuchen 16 und 36 mit 45 Gew.-Prozent Zeolith hergestellten Keramikschaumstoffe wurden auf Grund ihrer schlechteren Qualität für die weiteren Versuche nicht verwendet.

\subsection{Untersuchungen zur Schadstoffadsorption an Keramikschaumstoffen auf der Basis von Zeolithen}

Zielrichtung des Einsatzes der Keramikschaumstoffe sind die Schalldämpfung und die Eliminierung von Schadstoffen bei Verbrennungskraftmaschinen (4). Als Verbrennungsmotoren wurden als Modell zwei Serienmotoren aus den Skoda-Werken verwendet (Skoda 120 und Skoda Favorit $136 \mathrm{~L}$ ). An diese wurde in das Abgasrohr ein zusätzliches Rohrstiick mit dem eingepassten Keramikschaum angeschlossen. Die Länge des Keramikschaumstoffs betrug $100 \mathrm{~mm}$ bei einem Durchmesser von $65 \mathrm{~mm}$. Die Messungen wurden jeweils über einen Zeitraum von einer Stunde in Intervallen von fuinf Minuten durchgefuihrt, wobei die Motoren eine Drehzahl von $1500 \mathrm{~min}^{-1}$ hatten.

\begin{tabular}{|l|r|r|r|r|}
\hline ŠKODA 120 & $\begin{array}{r}\text { Interval of } \\
\text { measurements } \\
{[\mathrm{min}]}\end{array}$ & $\begin{array}{r}\mathrm{CO} \\
{[\%]}\end{array}$ & $\begin{array}{r}\mathrm{C}_{\mathrm{x}} \mathrm{H}_{\mathrm{y}} \\
{[\mathrm{ppm}]}\end{array}$ & $\begin{array}{r}\text { revs./min } \\
{\left[\mathrm{min}^{-1}\right]}\end{array}$ \\
\hline $\begin{array}{l}\text { Cold start } \\
\text { without filter }\end{array}$ & 5 & 7,57 & 843 & 638 \\
\hline $\begin{array}{l}\text { Warm motor } \\
\text { without filter }\end{array}$ & 5 & 8,66 & 920 & 673 \\
\hline $\begin{array}{l}\text { Warm motor } \\
\text { with cold filter }\end{array}$ & 5 & 6,61 & 517 & 667 \\
\hline $\begin{array}{l}\text { Warm motor, } \\
\text { warm filter }\end{array}$ & 5 & 7,57 & 492 & 252 \\
\hline $\begin{array}{l}\text { Warm motor, } \\
\text { warm filter }\end{array}$ & 5 & 7,76 & 534 & 710 \\
\hline
\end{tabular}

Tab. 5: Ergebnis der Messungen der Kohlenwasserstoffe und von Kohlenmonoxid beim Motor Skoda 120

\begin{tabular}{|l|r|r|r|r|r|r|r|}
\hline $\begin{array}{l}\text { ŠKODA } \\
136\end{array}$ & $\begin{array}{r}\text { Intervals } \\
\text { of } \\
\text { mesure- } \\
\text { ments } \\
{[\text { min] }]}\end{array}$ & $\begin{array}{r}\text { Temp. } \\
\text { of } \\
\text { exaust } \\
\text { gases } \\
{\left[{ }^{0} \mathrm{C}\right]}\end{array}$ & $\begin{array}{r}\text { Temp. } \\
\text { of en- } \\
\text { viron- } \\
\text { ment } \\
{\left[{ }^{0} \mathrm{C}\right]}\end{array}$ & $\begin{array}{r}\text { rev. } \\
\text { min }\end{array}$ & $\mathrm{CO}$ & $\mathrm{CO}_{2}$ & $\mathrm{C}_{\mathrm{x}} \mathrm{H}_{\mathrm{y}}$ \\
\hline $\begin{array}{l}\text { Cold motor } 1] \\
\text { without filter }\end{array}$ & 6 & 40 & 21 & 911 & 1,02 & 13,55 & 333 \\
\hline Cold filter & 6 & 30 & 21 & 899 & 0,77 & 11,87 & 298 \\
\hline Wppm]
\end{tabular}

Tab. 6: Ergebnisse der Messungen von Kohlenwasserstoffen und Kohlenmonoxid beim Motor Skoda Favorit $136 \mathrm{~L}$

Bei der Verbrennung in Motoren entstehen neben dem Hauptverbrennungsprodukt Kohlendioxid $\left(\mathrm{CO}_{2}\right)$ Kohlenmonoxid und Kohlenwasserstoffe unterschiedlicher Art (aliphatische Kohlenwasserstoffe aus der unvollständigen Verbrennung und PAK aus Zusätzen bzw. durch thermische Prozesse im Motorenraum) sowie Stickoxide (bei Temperaturen oberhalb $700^{\circ} \mathrm{C}$ aus den Elementen im Verbrennungsraum) (6). Die Mengen an Kohlenmonoxid 
und Kohlendioxid aus der Verbrennung in Abhängigkeit von der Art des Adsorbens sind in den Tabellen 5 und 6 dargestellt. Die Untersuchung der cyclischen Kohlenwasserstoffe erfolgte an Proben, die vom Keramikschaum desorbiert wurden. Ihre Zusammensetzung war folgende (HPLC):

Pyrene

Phenanthrene

Benzo(a)pyrene

$310,43 \mathrm{ppb}$

Benzo(a)anthracene

$2,5 \mathrm{ppb}$

$0,37 \mathrm{ppb}$

$31,4 \mathrm{ppb}$

Benzo(b)fluoranthene $\quad 11,7 \mathrm{ppb}$

Dibenzo(a,h)anthracene $\quad 2,09 \mathrm{ppb}$

Damit konnte nachgewiesen werden, dass zum einen die Zeolithe nach der thermischen Behandlung noch zur Adsorption von Schadstoffen befähigt sind und zum anderen die adsorbierte Menge an Kohlenmonoxid ca. $50 \%$, die der cyclischen Kohlenwasserstoffe ca. ein Drittel beträgt.

\section{Diskussion der Ergebnisse}

Auf der Basis von Recyclat-Polyolen, die zum einen aus PET und zum anderen aus PUR-Schaumstoffen durch Glykolyse erhalten wurden, konnten Formulierungen zur Herstellung von PUR-Hartschaumstoffen entwickelt werden, in die auf das Polymer bezogen bis zu 50 Gew.-Prozent an anorganischen Füllstoffen in Form natürlich vorkommender Zeolithe eingearbeitet werden konnten. Die mit Zeolithen gefuillten PUR-Hartschaumstoffe wurden verschiedenen Ausbrennprogrammen unterworfen, durch die die organische Schaumstoffmatrix abgebaut und in gasförmige Bestandteile umgewandelt wurde. Dadurch verbleibt ein rein anorganisches Gerüst, das durch eine erhöhte Temperatur seine Schaumstruktur behält. Die Temperatur zum Sintern der Zeolith-Teilchen beträgt nur $1100^{\circ} \mathrm{C}$ und liegt damit wesentlich unter den meisten gegenwärtig eingesetzten keramischen Materialien, aber mit einer Einsatztemperatur bis $950^{\circ} \mathrm{C}$ immer noch so hoch, dass die Keramikschaumstoffe in der Schalldämmung oder als Entgiftungsmatrices bei Verbrennungskraftmaschinen eingesetzt werden können. Der gesamte Ausbrennprozess dauert bei einem optimierten Temperaturprogramm fünf bis sechs Stunden zuzüglich der Abkuihlzeit. Unter Berücksichtigung der Tatsache, dass als Polymermatrix zu ca. 50 \% kostengüinstige Recyclingmaterialien und als keramisches Material ein natürlich vorkommendes Mineral verwendet werden und der Herstellungsprozess vergleichsweise einfach ist, steht mit diesem Verfahren und diesem Material eine kostengünstige Alternative zur Verfuigung. Wie die bisherigen Untersuchungen an Verbrennungskraftmaschinen gezeigt haben, behalten die gesinterten Zeolithe in der Schaumstruktur ihre Adsorptionsfähigkeit bei und adsorbieren ca. 50 \% der mit dem Abgasen transportierten Schadstoffe (5). Überraschend ist in diesem Zusammenhang, dass auch der Kohlenmonoxidanteil im Abgas um ca. $50 \%$ gesenkt wird. Wesentlich ist, dass polycyclische aromatische Kohlenwasserstoffe (PAK) zu einem großen Teil aus dem Abgas bereits bei relativ kleinen Filterlängen entfernt und an die Matrix gebunden werden.

\section{Anmerkungen/Literatur}

(1) H.-D. Hunger, G. Behrendt, R. Langenstraßen, K.-H. Schmidt: Poröse Sinterkeramikwerkstoffe und Verfahren zu ihrer Herstellung, DE-OS 19963554 (22.12.1999/05.07.2001)

(2) G. Behrendt, A. Lobeda, M. Pohl: Verfahren zur Herstellung von Polyesteralkoholen sowie Polyesteralkohole, DE Anm. 19817538.8 vom 16.04.1998, PCT WO 99/54380 (28.10.99)

(3) G. Behrendt, M. Pohl: Verfahren zur Herstellung von Polyolen und diese Polyole, DE Anm. 19817536.1 vom 19.04.1998, DE-OS 19917932 (21.10.99)

(4) M. Lorko, I. Pandova: The possibilities of zeolites utilisation on manufacture of the filters for the combustion engines exhaust systems, Manufacturing engineering, 1/2003

(5) M. Sucha, T. Gondova, I. Pandova, K. Florian, K. Reiffova, P. Oravec, J. Macala: The utilisation of zeolites for exhaust gases contaminant adsorption, Chemicke listy 96/2002.

(6) I. Pandova: The possibilities of zeolites for detoxikation of exhaust of the combustion engines, Acta Montanistica Slovaca, 6/2001

\section{Danksagung}

Die Autoren danken dem Internationalen Büro des BMBF fuir die finanzielle Unterstiitzung des Vorhabens. Weiter gilt der Dank der Autoren der Th. Goldschmidt AG für die kostenlose Überlassung der Stabilisatoren sowie Herrn Prof. Dr.-Ing. Tietgens für die Bestimmung der Korngrößenverteilungen der Zeolithe.

\section{Autoren}

Prof. Dr.-Ing. Martin Lorko Technickej univerzitet Košice Fakulta Vyrobnych Technologii Plzenska 10, SK-08001 Prešov, Slowakei

Telefon +421517723012

E-Mail: lorko.martin@fvt.sk

\section{Dr. Peter Oravec}

Technickej univerzitet Košice

Fakulta Vyrobnych Technologii

Plzenska 10, SK-08001 Prešov, Slowakei

Dipl.-Ing. (FH) Karlheinz Schmidt

Technische Fachhochschule Wildau

Fachbereich Ingenieurwesen/Wirtschaftsingenieurwesen

Telefon +493375 508-176

E-Mail: khschmid@igw.tfh-wildau.de

\section{Dr. sc. nat. Hans-Dieter Hunger}

Technische Fachhochschule Wildau

Fachbereich Ingenieurwesen/Wirtschaftsingenieurwesen

Telefon +493375 508-176

E-Mail: hdhunger@igw.tfh-wildau.de

\section{Prof. Dr. rer. nat. Gerhard Behrendt}

Technische Fachhochschule Wildau

Fachbereich Ingenieurwesen/Wirtschaftsingenieurwesen

Telefon +493375 508-591

E-Mail: behrendt@vt.tfh-wildau.de 